\title{
Psychological Interventions for Pediatric Headache Disorders: A 2021 Update on Research Progress and Needs
}

\author{
Kaelynn E. Knestrick ${ }^{1} \cdot$ Robert C. Gibler $^{1} \cdot$ Brooke L. Reidy $^{1,2} \cdot$ Scott W. Powers ${ }^{1,2,3}$ (1) \\ Accepted: 10 November 2021 / Published online: 2 February 2022 \\ (c) The Author(s), under exclusive licence to Springer Science+Business Media, LLC, part of Springer Nature 2022
}

\begin{abstract}
Purpose of Review This review summarizes key findings from recent investigations of psychological interventions for pediatric headache disorders and discusses important avenues for future research.

Recent Findings Cognitive Behavioral Therapy (CBT) is effective in reducing headache days among youth with chronic headache. There is mixed evidence for the benefit of CBT on reducing disability associated with migraine, suggesting that there is room to optimize CBT by leveraging complementary or alternative psychological interventions, such as Acceptance and Commitment Therapy (ACT) and mindfulness-based approaches. Tailoring CBT may be especially important for youth with more impairing or complex clinical presentations, such as those with continuous headache. Using eHealth and novel study designs to expand access to and dissemination of psychological interventions is promising.

Summary Although CBT is the gold standard psychological treatment for youth with migraine, we are only beginning to understand how and why it is effective. Other promising psychological treatments are available, and studies are beginning to examine how CBT can be optimized to fit the unique needs of each patient. Improving access and equitability of care for youth with migraine will require tailoring psychological treatments for patients with varying headache presentations and youth from a variety of cultural, racial, ethnic, and linguistic backgrounds.
\end{abstract}

Keywords Migraine $\cdot$ Headache $\cdot$ Treatment $\cdot$ Pediatric $\cdot$ CBT $\cdot$ Psychological interventions

\section{Introduction}

Headache and migraine are common chronic pain conditions among youth $[1,2]$, with migraine affecting about 10 percent of school-aged children and over $20 \%$ of older adolescents $[3,4]$. Youth with headaches frequently experience significant challenges in multiple domains of daily living, including school and social functioning [5]. Chronic headache can also

This article is part of the Topical Collection on Childhood and Adolescent Headache

Scott W. Powers

Scott.powers@cchmc.org

1 Division of Behavioral Medicine and Clinical Psychology, Cincinnati Children's Hospital Medical Center, Cincinnati, $\mathrm{OH}$, United States

2 Department of Pediatrics, University of Cincinnati College of Medicine, Cincinnati, $\mathrm{OH}$, United States

3 Headache Center, Cincinnati Children's Hospital Medical Center, Cincinnati, OH, United States be associated with psychiatric comorbidities such as depression and anxiety, which may confer an elevated risk for functional impairment and healthcare utilization $[6,7]$.

Although the current best-practice recommendations for the treatment of headache in youth emphasize a combined bio-behavioral approach that includes pill-based and psychological interventions [8], considerable evidence suggests that pharmacological treatment is not superior to placebo [9]. Our research team recently found that youth who participated in the Childhood and Adolescent Migraine Prevention (CHAMP) medication study maintained reductions in headache days and migraine-related disabilities three years after they participated in the trial, irrespective of whether they received pharmacotherapy or a placebo pill [10••]. Given that pill-based therapies can be associated with high rates of adverse events and side effects [11], the available data suggest that psychological interventions should be a front-of-the-line treatment option for pediatric migraine [7].

Evidence for the efficacy of psychological interventions for the treatment of headaches in youth has grown substantially over the past several decades [12•]. The current 
review summarizes recent developments in research examining the effectiveness of these interventions for pediatric headache disorders. We also highlight important directions for future research focused on understanding why psychological treatments work, optimizing and tailoring existing interventions to the needs of pediatric patients with migraine, investigating other promising psychological interventions for pain among youth with migraine, and increasing access to evidence-based care.

\section{Psychological Interventions for Pediatric Migraine: Cognitive Behavioral Therapy as the Gold Standard Treatment}

The leading and most frequently studied psychological intervention for the management of migraine in youth is cognitive-behavioral therapy (CBT; see Table 1 for a summary of treatment components for this intervention). Painfocused CBT teaches patients how to use and apply relaxation, behavioral, and cognitive coping skills to manage pain, reduce disability associated with headaches, and prevent headaches from starting or worsening. This intervention can also be helpful in reducing comorbid mental health symptoms, such as symptoms of anxiety and depression [13].

A 2018 Cochrane review of psychological therapies for the treatment of chronic pain in children and adolescents [12•] found that cognitive and/or behavioral interventions significantly reduced headache days and intensity across 15 randomized clinical trials (8 CBT, 7 behavioral strategies only). The number of intervention sessions needed for an additional beneficial outcome (NNTB) values were low in reviewed studies (between 2.59 and 3.58 sessions were needed to achieve a 50\% reduction in headache days), suggesting that treatment gains occurred relatively quickly. The review found less, but still positive, evidence for the benefit of psychological therapies on reducing headacherelated disability. Collectively, these results highlighted that non-medicine interventions for the treatment of migraine in youth are safe and effective, and called for additional controlled, high-quality intervention studies to optimize treatment outcomes and target headache-related disability and co-occurring mental health concerns among youth with migraine and other chronic pain conditions.

\section{Where to Go from Here: Optimizing and Increasing the Accessibility of Psychological Interventions}

\section{Understanding How and Why CBT is Effective for Youth with Migraine}

Although there is compelling evidence for CBT as a frontline intervention for youth with migraine, data that illustrate how and why this treatment is effective are only emerging. A recent study revealed that youth with migraine who received 8 sessions of CBT exhibited pre-to-post-treatment changes in prefrontal cortical (PFC) activity, with concomitant alterations in functional connectivity between the PFC and the

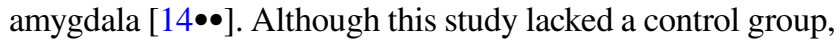
these preliminary findings suggest that CBT may produce

Table 1 Components of headache-focused cognitive-behavioral therapy (CBT)

\begin{tabular}{|c|c|c|}
\hline CBT Component & Explanation & Purpose \\
\hline Diaphragmatic breathing & $\begin{array}{l}\text { Slow the rate of breathing while engaging the diaphragm } \\
\text { muscle during inhalation and exhalation }\end{array}$ & $\begin{array}{l}\text { Decrease autonomic nervous system arousal associated } \\
\text { with pain and psychological distress }\end{array}$ \\
\hline Progressive muscle relaxation & Sequentially tense and relax major muscle groups & $\begin{array}{l}\text { Decrease muscle tension and autonomic arousal that } \\
\text { can contribute to pain }\end{array}$ \\
\hline Guided imagery & $\begin{array}{l}\text { Create a calming mental scene that utilizes primary } \\
\text { senses }\end{array}$ & $\begin{array}{l}\text { Decrease attention to pain, promote feeling of relaxation } \\
\text { using imagination }\end{array}$ \\
\hline Distraction & Intentionally engaging in valued and pleasant activities & $\begin{array}{l}\text { Decrease attention to pain and increase participation in } \\
\text { important activities despite pain }\end{array}$ \\
\hline Lifestyle Management & $\begin{array}{l}\text { Develop behavioral plans with patients and parents to } \\
\text { maintain regular intake of food and water, healthy } \\
\text { sleep practices, and regular exercise }\end{array}$ & $\begin{array}{l}\text { Promote optimal autonomic nervous system regulation } \\
\text { and prevention of headaches }\end{array}$ \\
\hline Activity Pacing & $\begin{array}{l}\text { Promote a balanced level of activity that incorporates } \\
\text { scheduled rest breaks into daily schedule }\end{array}$ & $\begin{array}{l}\text { Decrease the likelihood of headaches starting or } \\
\text { worsening with activity }\end{array}$ \\
\hline Thought Identification & $\begin{array}{l}\text { Identify negative or problematic thoughts associated } \\
\text { with pain or other symptoms }\end{array}$ & $\begin{array}{l}\text { Increase awareness of relationships among thoughts, } \\
\text { emotions, physical feelings, and behavior }\end{array}$ \\
\hline Cognitive Restructuring & $\begin{array}{l}\text { Discuss unhelpful thought patterns that can contribute to } \\
\text { worsening pain or distress, and/or introduce cognitive } \\
\text { strategies that challenge unhelpful thoughts or predictions }\end{array}$ & $\begin{array}{l}\text { Modify unhelpful patterns of thinking to identify } \\
\text { realistic and balanced alternatives that reduce pain } \\
\text { and distress, and promote optimal daily functioning }\end{array}$ \\
\hline
\end{tabular}


changes in neural functioning in brain regions responsible for cognitive control, emotional regulation, and pain modulation. The National Institute of Neurological Disorders and Stroke (NINDS) and the National Center for Complementary and Integrative Health (NCCIH) have funded an integrated mechanistic clinical study (R01 NS101321 \& R01 AT010071) that investigates neural alterations associated with specific components of CBT (e.g., cognitive restructuring techniques versus relaxation training) as well as a pill placebo. Results from this large mechanistic study will have the potential to lay the neurobiological foundation for the development of personalized CBT interventions and may provide more compelling evidence to providers and payors for why nonpharmacological interventions such as CBT should be a primary treatment approach for youth with migraine [15].

\section{Adapting and Supplementing CBT with Tailored Interventions to Improve Patient Outcomes}

As noted previously, the 2018 Cochrane review found mixed evidence for the benefit of CBT on reducing migrainerelated disability, particularly when treatment effects were examined at follow-up [12•]. Further optimization of available psychological interventions is needed, and focus should be placed on addressing the impact of headaches on the daily functioning and quality of life of children and adolescents. Although pain-focused CBT is effective, it is not perfect and may not fully meet the needs of some youth who present to a specialty headache center for care.

Certainly, if comorbid mental health symptoms (e.g., depression, post-traumatic stress) are acute and exacerbating current pain symptomology, cognitive-behavioral and/ or trauma-informed interventions should be prioritized that directly address these concerns. Additionally, patients' clinical presentations may influence how pain-focused CBT should be tailored or supplemented to promote treatment success. For example, a treatment naïve patient referred directly to a headache center from primary care and a tertiary referral patient who has tried multiple pharmacological (or other non-pharmacological) treatments without success may look very different in terms of their beliefs about pain, communication with providers, and treatment expectancies. Similarly, a patient who experiences 5 to 6 headache days per month may have different nonpharmacological treatment needs than youth with continuous headache (who may either have New Daily Persistent Headache or unremitting headache resulting from chronified migraine) that has persisted for several months [16•].

Taking a patient and family-centered approach to treating highly disabled patients and/or patients with more complex headache treatment histories is critical to increasing both buy-in for psychological headache treatments and expectations of individual treatment success in pain-focused CBT [17]. Motivational interviewing (MI) is a collaborative, patient-centered approach to interacting with patients that have been used to promote health-related behavior change among youth with chronic medical conditions [18], can be used in conjunction with other therapies [19], and may be particularly helpful in promoting readiness for engagement in psychological treatment for migraine. MI can be utilized by psychologists or other healthcare providers. Among youth with chronic medical conditions, MI interventions have been shown to increase conversation length between providers and patients, which in turn is associated with improved adherence to medical recommendations [20]. Taking a MI stance in the context of providing pain education may also optimize this component of CBT for headache. Future research should focus on determining the added benefits of interventions like MI in clinical outcomes when combined with cognitivebehavioral interventions for youth with migraine [21].

\section{Examining the Potential Benefits of Third-wave Psychological Therapies}

"Third wave" psychological approaches, such as mindfulness or Acceptance and Commitment Therapy (ACT), may also be useful interventions for addressing significant headacherelated disability among youth who are better suited to more cognitive and/or meta-cognitive pain management strategies. Mindfulness involves bringing attention to the present moment while allowing uncomfortable thoughts and physical sensations to come and go without judgment and direct intervention [22]. Mindfulness and acceptance-based approaches are distinct from cognitive-behavioral frameworks in their emphasis on increasing participation in valued activities despite the presence of pain, as opposed to reducing pain and associated symptoms.

Mindfulness-based interventions, such as MindfulnessBased Cognitive Therapy (MBCT), are effective in reducing pain intensity and disability among adults with a variety of pain conditions [23] and can be effective in reducing functional disability [24] and increasing pain acceptance among youth with chronic pain [25]. A recent phase II trial of MBCT tailored for the treatment of migraine in adults revealed statistically significant reductions in migraine-related disability in the active treatment versus the control group, although no group differences in self-reported headache days or pain intensity were found [26•]. To our knowledge, no clinical trials of mindfulness-based interventions have been conducted in a pediatric headache sample, though pilot trials are underway [27]. In contrast, the evidence base for ACT in the treatment of chronic pain in children and adolescents is growing, suggesting that this intervention may hold promise for use among youth with headache conditions [28]. Complementary and alternative treatments such as acupuncture, aromatherapy, and 
hypnosis also have some research support and have also been successfully incorporated in multidisciplinary headache clinics [29]. Rigorous clinical research effort is needed to expand our understanding of the benefits of these interventions for youth with migraine.

\section{Moving Toward a Diverse, Equitable and Inclusive Scientific and Clinical Practice}

It is also imperative that our interventions evolve in a manner that will meet the needs of patients from all racial, ethnic, socioeconomic, and language backgrounds. One limitation of the current state of our science relates to sampling in rigorous controlled trials. Migraine is a genetic disease with high prevalence rates across populations, and the prevalence of migraine in men is not negligible [30]. Yet, the majority of participants included in clinical trials are White, English speaking females [31]. Although this demographic breakdown is generally representative of treatment-seeking patients with migraine, important questions remain about the systemic barriers in health care that lead to these discrepancies, as well as other sources of potential bias that can affect recruitment efforts and participation in clinical trials. A clear avenue to increase the generalizability of controlled trials is to purposefully recruit individuals from traditionally underrepresented and underserved backgrounds ensure that samples represent the populations for whom treatments are intended. Still, improving the representativeness of samples in clinical trials is only one piece to the broader challenge our field faces in our efforts to understand why and for whom psychological interventions for pediatric migraine are effective.

Moving toward more equitable care in clinical practice and research will require ongoing adaptation of existing psychological interventions to reflect diversity in patient populations while maintaining the core principles of treatment. Because beliefs about pain and the best ways to manage pain can vary considerably across racial and ethnic groups, taking time to understand how patients view their pain experience will allow providers to share a rationale for treatment that is consistent with these beliefs, and may enhance patient engagement in a psychological intervention [32]. Recognizing the need to "meet patients where they are" in treatment may also assist researchers in identifying outcome variables that have historically been neglected but represent an important aspect of treatment expectations for patients from diverse backgrounds.

\section{Extending the Reach of CBT: Developing and Testing Novel eHealth and $m$ Health Interventions}

Treatment accessibility also remains a barrier to broader utilization of evidence-based care. In clinic settings, limited availability of providers with training and expertise in behavior change represent challenges to providing youth with evidence-based headache management strategies [33, 34]. CBT provided by professionals with training in behavioral medicine is not widely available to youth who do not live in close proximity to a pediatric hospital, and the frequency of CBT treatment sessions can be burdensome for many families [35]. That said, the COVID-19 pandemic has forced healthcare providers to leverage technology to offer services, and it is likely that this will continue for the foreseeable future as telemedicine becomes an increasingly preferred mode of treatment delivery.

Digital therapeutic tools such as smartphone apps and internet-delivered CBT interventions have also been developed to increase accessibility of evidence-based behavioral headache management strategies, although the majority of pilot and/or controlled studies have been conducted in adult populations [34]. Recently, a randomized trial conducted among youth with chronic pain (including chronic headache) demonstrated that the Web-based Management of Adolescent Pain (WebMAP) program, a cognitive-behavioral pain management intervention delivered through an interactive website [36] and accompanying mobile app (i.e., WebMAP Mobile), was superior to usual care in increasing participants' impressions of change post-treatment and at a three month follow-up [37•]. In contrast, a pilot randomized trial of a smartphone app delivered in conjunction with eight weeks of relaxation-based biofeedback treatment did not produce statistically significant reductions in headache frequency among adolescents with migraine [38].

Additional research is needed to identify standalone digital interventions that are efficacious for youth with migraine. Although myriad mobile headache management apps are available for public use, the vast majority do not incorporate evidence-based behavior change techniques and headache self-management strategies and have not been tested in randomized controlled trials [39]. Recently, Hommel and colleagues [40••] developed Migraine Manager, a smartphone app which includes migraine-specific self-management strategies in addition to generalized evidence-based coping skills. In a small open-arm pilot feasibility trial, adolescents who received Migraine Manager reported statistically significant reductions in headache days and physical functioning quality of life. Usage data showed that participants engaged regularly with app content and most frequently completed self-management skills such as setting goals and strategies to manage school challenges. The effect of Migraine Manager on headache frequency, quality of life, and migraine-related disability is currently being tested in a randomized clinical trial (R01 NR019426-01). Rigorous data supporting the effectiveness of a migraine-specific self-management app may increase provider confidence in "prescribing" eHealth platforms as an adjunct to traditional face-to-face CBT. 


\section{Leveraging Innovative Study Designs to Increase Accessibility to Care}

Cognitive-behavioral coping skills for youth with migraine are effective when practiced [41]; unfortunately, research has shown that adherence with home practice recommendations can be low in both youth and adults receiving CBT [42], with poor adherence being a predictor of poorer treatment outcomes [43]. In addition to adherence once recommended, the 2012 National Health Interview Survey found that of the 2,795 children included with one or more pain-related condition, only about $3 \%$ reported using any mind and body skills for self-management [44]. Given this, the National Center for Complementary and Integrative Health (NCCIH) recently funded a multisite Mind Body Balance for Pediatric Migraine project (U01 AT010132) that will evaluate different levels of treatment components that comprise a nurse delivered relaxation-based intervention (i.e., duration of intervention, format of home practice support, amount of follow-up adherence prompting), to find an optimized intervention that can be delivered by specially trained healthcare professionals that are already a part of the child's headache treatment team. The Multiphase Optimization Strategy (MOST) [45] allows for the development of treatment packages that are efficient, cost effective, and can be made more widely available, and can be used to increase accessibility of efficacious interventions for youth with migraine.

Recently, group-based nonpharmacological interventions have also been developed to address common challenges youth with chronic pain experience in daily life. For example, the FIT Teens study is an ongoing, group-based clinical trial that is evaluating the efficacy of a novel, integrated CBT and neuromuscular training protocol for juvenile fibromyalgia [46]. Initially developed to address the difficulties youth with JFM experience with integrating daily exercise recommendations into their routines, the FIT Teens intervention has shown promising initial results in reducing functional disability [47]; participants and trainers both emphasize benefits of the group training format in facilitating treatment engagement [48]. Future studies should examine the feasibility of group-based psychological interventions for youth with migraine, as well as treatment adherence and outcomes in this context.

\section{Conclusion}

Psychological interventions such as CBT are safe and effective treatments for pediatric pain management and should be considered a frontline treatment for youth with migraine. In the coming years, the field may have some answers for the question "Why does CBT work for kids and teens with migraine?" These insights, along with tailored patient and family-centered interventions, will lay the groundwork for the development of personalized nonpharmacological interventions that consider each child's unique needs. Leveraging the potential of mindfulness-, acceptance and commitment-, and group-based interventions, as well as digital technology may improve existing treatments and expand access to evidence-based care. In order to make a broader public health impact, research that includes diverse samples of children and adolescents with headaches will be desperately needed in the coming years to ensure the equitability and accessibility of nonpharmacological interventions to all youth with headache disorders. Additional research that optimizes nonpharmacological intervention packages that can be delivered by a variety of healthcare professionals will also facilitate increased access to evidence-based migraine treatment.

Acknowledgements The authors gratefully acknowledge Susan LeCates, MSN, APRN, CNP for reviewing and providing feedback on this manuscript.

Funding This work was supported by an R01 (R01AT010171) and U01 (U01 AT010132) grant from the National Center for Complementary and Integrative Health, an R01 grant (R01NS101321) from the National Institute of Neurological Disorders and Stroke, and a training grant from the National Institute of Diabetes and Digestive and Kidney Diseases (T32DK063929).

\section{Compliance with Ethical Standards}

Conflict of Interest The authors declare that they have no conflict of interest.

Human and Animal Rights and Informed Consent This article does not contain any studies with human or animal subjects performed by any of the authors.

\section{References}

Papers of particular interest, published recently, have been highlighted as:

- Of importance

$\bullet$ Of major importance

1. King S, et al. The epidemiology of chronic pain in children and adolescents revisited: A systematic review. Pain. 2011;152(12):2729-38.

2. Powers SW, Coffey CS, Hershey AD. Treatment of pediatric migraine. N Engl J Med. 2017;376(14):1388-9.

3. Abu-Arefeh I, Russell G. Prevalence of headache and migraine in schoolchildren. BMJ. 1994;309(6957):765-9.

4. Split W, Neuman W. Epidemiology of migraine among students from randomly selected secondary schools in Lodz. Headache. 1999;39(7):494-501.

5. Gorodzinsky AY, Hainsworth KR, Weisman SJ. School functioning and chronic pain: A review of methods and measures. J Pediatr Psychol. 2011;36(9):991-1002.

6. Bellini B, et al. Headache and comorbidity in children and adolescents. J Headache Pain. 2013;14(1):79. 
7. Law EF, et al. Economic impact of headache and psychiatric comorbidities on healthcare expenditures among children in the United States: A retrospective cross-sectional study. Headache. 2019;59(9):1504-15.

8. Powers SW, et al. Cognitive behavioral therapy plus amitriptyline for chronic migraine in children and adolescents: A randomized clinical trial. JAMA. 2013;310(24):2622-30.

9. Le K, et al. Is topiramate effective for migraine prevention in patients less than 18 years of age? A meta-analysis of randomized controlled trials. J Headache Pain. 2017;18(1):69.

$10 . \bullet$ Powers SW, et al. Prevalence of headache days and disability 3 years after participation in the childhood and adolescent migraine prevention medication trial. JAMA Netw Open. 2021;4(7):e2114712. Follow-up analysis from CHAMP medication trial demonstrating that participants maintained a reduction in headache days three years after participation irrespective of whether they received pharmacotherapy or placebo.

11. Powers SW, et al. Trial of amitriptyline, topiramate, and placebo for pediatric migraine. N Engl J Med. 2017;376(2):115-24.

12. Fisher E, et al. Psychological therapies for the management of chronic and recurrent pain in children and adolescents. Cochrane Database Syst Rev. 2018;9(9):Cd003968. Systematic review and meta-analysis examining the effectiveness of psychological interventions for pediatric chronic pain conditions, including headache.

13. Ernst MM, O'Brien HL, Powers SW. Cognitive-behavioral therapy: How medical providers can increase patient and family openness and access to evidence-based multimodal therapy for pediatric migraine. Headache. 2015;55(10):1382-96.

14. $\bullet$ Nahman-Averbuch, $\mathrm{H}$, et al. Alterations in brain function after cognitive behavioral therapy for migraine in children and adolescents. Headache. 2020;60(6):1165-1182. Pilot study examining changes in neural functioning among youth with migraine following participation in cognitive-behavioral therapy.

15. Nahman-Averbuch $\mathrm{H}$, et al. The promise of mechanistic approaches to understanding how youth with migraine get better. An Editorial to the 2020 Members' Choice Award Paper. Headache. 2021;61(6):803-804.

16. Reidy BL, et al. Clinic-based characterization of continuous headache in children and adolescents: Comparing youth with chronic migraine to those with new daily persistent headache. Cephalalgia. 2020;40(10):1063-1069. Examination of a specific subgroup of youth with chronic migraine whose headaches occur daily.

17. Tomlinson RM, Cousins LA, McMurty CM, Cohen LL. The power of pain self-efficacy: Applying a positive psychology framework to pediatric pain. Pediatr Pain Lett. 2017; 19(1):9-13.

18. McNeil DW, Addicks SH, Randall CL. Motivational interviewing for health behavior change. 2017; Oxford Handbooks Online.

19. Bricker J, Tollison S. Comparison of motivational interviewing with acceptance and commitment therapy: A conceptual and clinical review. Behav Cogn Psychother. 2011;39(5):541-59.

20. Nijs $\mathbf{J}$, et al. Integrating motivational interviewing in pain neuroscience education for people with chronic pain: A practical guide for clinicians. Phys Ther. 2020;100(5):846-59.

21. Reinauer $\mathrm{C}$, et al. Efficacy of motivational interviewing to improve utilization of mental health services among youths with chronic medical conditions: A cluster randomized clinical trial. JAMA Netw Open. 2021;4(10):e2127622.

22. Keng SL, Smoski MJ, Robins CJ. Effects of mindfulness on psychological health: A review of empirical studies. Clin Psychol Rev. 2011;31(6):1041-56.

23. Veehof MM, et al. Acceptance- and mindfulness-based interventions for the treatment of chronic pain: A meta-analytic review. Cogn Beh Ther. 2016;45(1):5-31.
24. Waelde LC, et al. A pilot study of mindfulness meditation for pediatric chronic pain. Children (Basel). 2017; 4(5).

25. Ruskin DA, et al. A mindfulness program adapted for adolescents with chronic pain: Feasibility, acceptability, and initial outcomes. Clin J Pain. 2017;33(11):1019-29.

26. Seng EK, et al. Does mindfulness-based cognitive therapy for migraine reduce migraine-related disability in people with episodic and chronic migraine? A phase $2 \mathrm{~b}$ pilot randomized clinical trial. Headache. 2019;59(9):1448-1467. Mindfulnessbased intervention trial for adults with migraine.

27. Sansone E, et al. Mindfulness meditation for chronic migraine in pediatric population: A pilot study. Neurol Sci. 2018;39(Suppl 1):111-3.

28. Pielech M, Vowles KE, Wicksell R. Acceptance and commitment therapy for pediatric chronic pain: Theory and application. Children (Basel). 2017;4(2).

29. Esparham A, et al. Pediatric headache clinic model: Implementation of integrative therapies in practice. Children (Basel). 2018;5(6).

30. Pressman A, et al. Prevalence of migraine in a diverse communityelectronic methods for migraine ascertainment in a large integrated health plan. Cephalalgia. 2016;36(4):325-34.

31. Robbins NM, Bernat JL. Minority representation in migraine treatment trials. Headache. 2017;57(3):525-33.

32. Campbell CM, Edwards RR. Ethnic differences in pain and pain management. Pain Manag. 2012;2(3):219-30.

33. Stubberud A, Linde M. Digital technology and mobile health in behavioral migraine therapy: A narrative review. Curr Pain Headache Rep. 2018;22(10).

34. Minen MT, et al. Electronic behavioral interventions for headache: A systematic review. J Headache Pain. 2016; 17.

35. Bekker MJ, Griffiths KM, Barrett PM. Improving accessibility of cognitive behavioural therapy for children and adolescents: Review of evidence and future directions. Clin Psychol. 2017;21(3):157-64.

36. Palermo TM, et al. Internet-delivered cognitive-behavioral treatment for adolescents with chronic pain and their parents: A randomized controlled multicenter trial. Pain. 2016;157(1):174-85.

37. Palermo TM, et al. A digital health psychological intervention (WebMAP Mobile) for children and adolescents with chronic pain: Results of a hybrid effectiveness-implementation steppedwedge cluster randomized trial. Pain. 2020;161(12):27632774. Study examining the effectiveness of a mobile health intervention for treatment of pediatric chronic pain.

38. Stubberud A, et al. Self-administered biofeedback treatment app for pediatric migraine: A randomized pilot study. Brain Beh. 2021;11(2).

39. Gamwell KL, et al. Systematic evaluation of commercially available pain management apps examining behavior change techniques. Pain. 2021;162(3):856-65.

40.• Hommel KA, et al. Digital therapeutic self-management intervention in adolescents with migraine: Feasibility and preliminary efficacy of "Migraine Manager". Headache 2020;60(6):11031110. Study examining the initial efficacy and feasibility of a migraine-specific self-management app designed for use among adolescents.

41. Van Diest AMK, et al. Treatment adherence in child and adolescent chronic migraine patients. Clin J Pain. 2017;33(10):892-8.

42. Tang W, Kreindler D. Supporting homework compliance in cognitive behavioural therapy: Essential features of mobile apps. JMIR Mental Health. 2017;4(2):e20.

43. Helbig S, Fehm L. Problems with homework in CBT: Rare exception or rather frequent? Behav Cogn Psychother. 2004;32(3):291-301.

44. Groenewald CB, et al. Complementary and alternative medicine use by children with pain in the United States. Acad Pediatr. 2017;17(7):785-93.

45. Collins LM, Murphy SA, Strecher V. The Multiphase Optimization Strategy (MOST) and the Sequential Multiple Assignment 
Randomized Trial (SMART): New methods for more potent eHealth interventions. Am J Prev Med. 2007;32(5 Suppl):S112-8.

46. Kashikar-Zuck S, et al. Randomized clinical trial of Fibromyalgia Integrative Training (FIT teens) for adolescents with juvenile fibromyalgia - Study design and protocol. Contemp Clin Trials. 2021;103:106321.

47. Kashikar-Zuck S, et al. Pilot randomized trial of integrated cognitivebehavioral therapy and neuromuscular training for juvenile fibromyalgia: The FIT Teens Program. J Pain. 2018;19(9):1049-62.
48. Kashikar-Zuck S, et al. A qualitative examination of a new combined cognitive-behavioral and neuromuscular training intervention for juvenile fibromyalgia. Clin J Pain. 2016;32(1):70-81.

Publisher's Note Springer Nature remains neutral with regard to jurisdictional claims in published maps and institutional affiliations. 